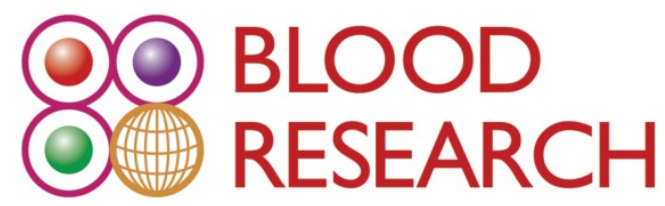

\title{
Induction-related mortality in adolescents and young adults with acute lymphoblastic leukemia in a resource-limited setting: do treatment-related complications create more impact than disease biology?
}

Sergio I Inclan-Alarcon, Santiago Riviello-Goya, Kevin Teran-De-la-Sancha, Oscar M Fierro-Angulo, Aldo A Acosta-Medina, Roberta Demichelis-Gomez, Christianne Bourlon

Citation: Inclan-Alarcon SI, Riviello-Goya S, Teran-De-la-Sancha $K$, et al. Induction-related mortality in adolescents and young adults with acute lymphoblastic leukemia in a resource-limited setting: do treatment-related complications create more impact than disease biology? Blood Res 2022. [Epub ahead of print]

Publisher's disclaimer: This manuscript has completed peer review and has been accepted for publication. We are distributing the authors' version of manuscript prior to technical and English editing, typesetting and proof reading. A final version approved by the authors' will follow in a regular issue of BLOOD RESEARCH. All legal disclaimers that apply to BLOOD RESEARCH also pertain to this production process. 
Induction-related mortality in adolescents and young adults with acute lymphoblastic leukemia in a resource-limited setting: do treatment-related complications create more impact than disease biology?

Sergio I Inclan-Alarcon ${ }^{1}$, Santiago Riviello-Goya ${ }^{1}$, Kevin Teran-De-la-Sancha ${ }^{1}$, Oscar M Fierro-Angulo ${ }^{2}$, Aldo A Acosta-Medina ${ }^{1}$, Roberta Demichelis-Gomez ${ }^{1}$, Christianne Bourlon ${ }^{1}$

${ }^{1}$ Department of Hematology and Oncology, ${ }^{2}$ Department of Medicine, Instituto Nacional de Ciencias Médicas y Nutrición Salvador Zubirán, Mexico City, Mexico

Correspondence to: Christianne Bourlon M.D.

Department of Hematology and Oncology, Instituto Nacional de Ciencias Médicas y Nutrición Salvador Zubirán, Vasco de Quiroga 15, Belisario Domínguez Sección XVI, Tlalpan, 14080, Mexico City, Mexico E-mail: chrisbourlon@hotmail.com 


\begin{abstract}
Background: Acute lymphoblastic leukemia (ALL) is a malignant clonal bone marrow disorder with a high mortality rate during the initial therapy. This retrospective study aimed to describe and analyze the risk factors and causes of induction-related mortality (IRM) in an adolescent and adult ALL population treated in a low- and middle-income country.
\end{abstract}

Methods: From 2009 to 2016, a total of 167 patients were included, of which 50.9\% were male with a median age of 28 years. B-immunophenotype represented $97.6 \%$, and high-risk cytogenetics were present in $23.3 \%$. During induction therapy, $91 \%$ had at least 1 complication, most of which were infectious, with an IRM of $12 \%$.

Results: Factors associated with increased mortality rate were central nervous system (CNS) status [CNS-3: hazard ratio (HR) 3.029; 95\% confidence interval (CI), 0.79-11.49; $P=0.103$ and CNS-2: HR, 9.98; 95\% CI, 2.65-37.65; $P=0.001]$ and dialysis requirement (HR, 9.15; 95\% CI, 2.44-34.34; $P=0.001)$.

Conclusion: Our study confirms that ALL patients treated in resource-constrained settings have high rates of IRM, mainly attributed to advanced disease and high tumor burden at diagnosis.

Key Words: Risk factors, Treatment outcome, Chemotherapy induced mortality, Precursor cell lymphoblastic leukemia-lymphoma, Developing Countries 


\section{INTRODUCTION}

Acute lymphoblastic leukemia (ALL) is characterized by a malignant transformation and subsequent clonal expansion of lymphoid progenitors, associated with various genetic and molecular abnormalities [1]. Diagnostic and therapeutic advances over the last decades have enabled highincome countries to achieve first complete remission (CR1) rates of $>80 \%$, improvements in overall survival (OS), and a decreased treatment- and induction-related mortality (IRM) [2]. Conversely, compared with the $<5 \%$ IRM reported in these settings, in low- and middle-income countries (LMICs), such as Mexico and Brazil, it remains as high as $17 \%$ to $26 \%$ [3-5].

Differences in clinical outcomes between adult and pediatric populations have been attributed to different first-line therapeutic regimens and to the favorable disease biology amongst children [6]. Adolescents and young adults (AYAs) are a particular subgroup facing important management challenges due to their transitioning position and increased likelihood of presenting with high-risk ALL features. Compared to that of children, AYAs have a greater incidence of Philadelphia chromosome positivity $(\mathrm{Ph}+)$, Philadelphia-like, and BCL2/MYC rearrangements, and decreased frequency of hyperdiploidy and ETV6/RUNX1-positivity [7]. Reports on long-term clinical outcomes have shown a decreased 5-year OS with increased mortality in CR1 for AYAs, mostly due to severe infectious diseases and IRM [8].

The following are known risk factors for IRM: male sex, T-cell ALL, high risk ALL, low platelet count and white blood cell count $>100,000$ at diagnosis, and patients with longer travel time to the clinic $[9,10]$. However, all this information comes exclusively from pediatric cohorts. To address the paucity of literature on AYAs and adults in LMICs, this study aimed to determine the cohort rates, causes, and risk factors of IRM. 


\section{MATERIALS AND METHODS}

We conducted a retrospective study including consecutive patients aged $\geq 16$ years with newly diagnosed ALL at our institution from January 2009 to September 2016. Patients with incomplete clinical records, those that received induction therapy prior to referral to our center, and those lost to follow-up before day 60 after induction were excluded. The study was approved by our center's Institutional Review Board (HEM-3679-21-22-1).

Clinical, laboratory, and outcomes data were collected from the physical and/or electronic medical records. Patients were classified as AYAs if their age was $\leq 40$ years. Socioeconomic status (SES) was defined according to monthly household income as follows: low-SES, $<180.00$ USD; middle-SES, $\geq 180.00$ USD; and high-SES, patients with private health insurance coverage. Performance status (PS) was stratified according to the Eastern Cooperative Oncology Group (ECOG) Performance Scale [11], and records were obtained at the time of ALL diagnosis.

ALL diagnosis was made in accordance with the 2016 WHO Classification of Tumors of Hematopoietic and Lymphoid Tissues [12]. A high-risk cytogenetic profile was defined as the presence of the following: translocation $\mathrm{t}(9,22)(P h+$ positive), mixed-lineage leukemia 1 (MLL1) rearrangements, hypodiploidy, and/or complex karyotypes. Central nervous system (CNS) status was defined as CNS-1, -2 , or -3 according to the NCCN Guidelines [13]. Based on previously published protocols, treatment regimens were grouped into Hyper-CVAD [14], our institutional protocol (HOP 0195/0612) [15], and other regimens.

IRM was defined as death within 60 days after the initiation of the induction regimen, during post-chemotherapy myelosuppression period, and not being associated with disease refractoriness or progression. Cause of death was defined as infectious if it was directly associated with septic shock or other complications related to an infectious disease, hematologic if it was attributed to major 
hemorrhage or thrombotic events, or other when it was none of the above. Overall survival (OS) was defined in days, considering the time from diagnosis to death or last follow-up.

Complications were reported as infectious, metabolic, or hematologic. Infectious complications included bloodstream, urinary tract, and skin and soft tissue infections, as well as pneumonia, invasive fungal infections, and C. difficile colitis. Metabolic complications comprised hipertransaminasemia, renal impairment with or without dialysis requirement, and tumor lysis syndrome. Major hemorrhagic or thrombotic events and disseminated intravascular coagulation (DIC) were considered hematologic complications. All complications included in the variables were those that developed between day 1 and 60 of induction treatment.

\section{Statistical analyses}

Qualitative data were described in terms of frequencies and percentages, and quantitative variables were described in terms of median and range. Mortality curves were constructed via the Kaplan-Meier method, and univariate survival analysis was performed using the log-rank test. Cox regression was performed for multivariable analysis. A $P$-value $\leq 0.05$ was considered significant for all statistical tests. All calculations were performed with SPSS version 25 (IBM Corp, Armonk, NY).

\section{RESULTS}

A total of 167 patients were included in the study. The median age of the cohort was 28 years (range, 16-70), 50.9\% $(\mathrm{N}=85)$ were male, and AYA represented $67.1 \%(\mathrm{~N}=112)$ of the cases. An ECOG $\leq 2$ was observed in $96.4 \%(\mathrm{~N}=161)$, and $48.5 \%(\mathrm{~N}=81)$ of patients had 1 or more additional comorbidities at diagnosis; of these $21 \%(\mathrm{~N}=17)$ had diabetes, $16.1 \%(\mathrm{~N}=13)$ had hypertension, and 44.4\% ( $\mathrm{N}=36)$ were obese. Regarding ALL diagnosis, immunophenotype was B-cell in 97.6\% $(\mathrm{N}=163)$ and $\mathrm{T}$-cell in $2.4 \%(\mathrm{~N}=4)$; high-risk cytogenetics were present in $23.3 \%(\mathrm{~N}=39)$. Additional baseline characteristics are presented in Table $\mathbf{1}$. 
Induction therapy with Hyper-CVAD was administered in $65.3 \%(\mathrm{~N}=109)$ of patients, HOP0195/0612 in 28.7\% ( $=48)$, and other regimens in 6\% $(\mathrm{N}=10)$. Flow cytometry demonstrated CD20 positivity in $68.9 \%(\mathrm{~N}=115)$ of cases. A total of 24 patients $(20.9 \%)$ were receiving rituximab in addition to chemotherapy. The median follow-up was 10 months (range, 10-108). All patients underwent complete remission assessment, and 77.2\% (N=129) achieved CR1. Among CD20-positive patients, $79.2 \%(\mathrm{~N}=19)$ of those receiving rituximab reached $\mathrm{CR} 1$ as compared to $76.9 \%(\mathrm{~N}=70)$ of those who were not receiving rituximab $(P=0.55)$. There was no difference in CR1 rates when analyzed by age group $(P=0.71)$ or cytogenetic risk stratification $(P=0.50)$.

Induction-related complications were reported in 152 (91\%) patients. The most common type of complications were infectious diseases in $87.4 \%(\mathrm{~N}=146)$ of patients, followed by metabolic complications in $46.1 \%(\mathrm{~N}=70)$, and hematologic in $11.1 \%(\mathrm{~N}=18)$. Complication types by subgroup are further described in Table 2. Regarding infectious complications, a bacterial isolate was obtained in $69.9 \%(\mathrm{~N}=102)$ of cases, most of which were gram-negative rods $(\mathrm{N}=72)$ followed by grampositive cocci $(\mathrm{N}=20)$. Invasive fungal infections (IFIs) represented 15 cases of Aspergillus sp. and 14 of Candida sp. A total of 46 patients developed shock during the course of induction therapy, and $85.7 \%(\mathrm{~N}=39)$ was related to sepsis.

IRM in our cohort was $12 \%(\mathrm{~N}=20)$. The cause of death was an infectious complication in 14 patients (77.8\%), a hemorrhagic complication in 3 patients $(16.7 \%)$, and 3 patients $(16.7 \%)$ had other causes of death. On univariate analysis, the factors related to a decreased OS after induction included the following: CNS status at diagnosis $(P<0.001)$, TLS $(P=0.005)$, DIC $(P=0.037)$, shock $(P<0.001)$, bloodstream infection $(P=0.020)$, requirement of renal replacement therapy $(P<0.001)$, and requirement of assisted invasive mechanical ventilation $(P<0.001)$. The presence of comorbidities, including diabetes, hypertension, and obesity, were not associated with an increased IRM $(P=0.49)$ (Table 3). The IRM of AYAs and older adults was $11.6 \%$ vs. $12.7 \%(P=0.83)$, respectively, with no difference in the causes of death. According to cytogenetics, there were no differences in mortality between the groups of normal karyotype, $\mathrm{Ph}+$, complex karyotype, and other abnormalities $(11.1 \%$ 
vs. $4.3 \%$ vs. $16.7 \%$ vs. $6.7 \%$, respectively; $P=0.68$ ). Leukocytosis, considering cutoff values according to leukemia phenotype, did not affect IRM (15.8\% vs. $11.0 \% ; P=0.43)$.

The multivariate analysis factors that remained significant for an increased IRM were CNS status (CNS-3: HR, 3.029; 95\%CI, 0.79-11.49; $P=0.103$ and CNS-2: HR, 9.98; 95\%CI, 2.65-37.65; $P=0.001)$ and dialysis requirement (HR, 9.15; 95\% CI, 2.44-34.34; $P=0.001)$ (Fig. 1, Table 3).

\section{DISCUSSION}

Current reports on survival outcomes for ALL have particularly focused on long-term mortality of patients from developed nations and pediatric cohorts; thus, information on IRM and its risk factors is scarce. In this setting, adults in LMICs are particularly faced with challenges associated with limitations in infrastructure and resources that hinder accessibility to molecular diagnosis, pediatric-inspired regimens, and supportive measures required for appropriate patient management during the post-induction period. To the best of our knowledge, this is the first report evaluating mortality and risk factors for IRM in an adult population living in a LMIC.

Our cohort's baseline demographics closely resemble those of other Latin American countries in terms of age distribution, CNS status, and PS, where a young median age and a high PS at diagnosis are expected. Patients in the AYA group, which in this study comprised more than $50 \%$, have been related with a worse prognosis. This is mainly attributed to the biology of disease, when compared with other age groups. Particularly in our group, where the prevalence of comorbidities was high, we had a low SES, and 25\% of high risk cytogenetics showed no impact on IRM rates [16].

In this cohort, we report an IRM of $12 \%$ and a CR1 of $77.2 \%$, which are within the reported rates from other ALL groups [14]. No significant difference for IRM or CR1 was observed when comparing the different induction regimens used, including those in which rituximab was added because of CD20 positivity. These findings are consistent with those of published reports, which 
shows improved disease-free survival and CR1 duration with rituximab use, but no direct impact on short-term outcomes such as those evaluated in the present study [17].

As expected for an LMIC, we report a high rate of complications during the induction therapy, where infection is the most common. Infections with proven bacterial foci and neutropenic fever were similar to those reported in pediatric and adult cohorts with the use of intensive regimens $[5,18]$. Remarkably, the prevalence of IFI in our cohort was significantly higher than expected, closely resembling IFI trends observed in patients with myeloid malignancies [19-21]. The explanation to this was most likely multifactorial and associated with the living situation of patients, limited spectrum of prophylactic antifungals, and renovations on hospital grounds. Cognizance is raised to the fact that IFIs did not significantly increase IRM, yet the long-term impact on clinical outcomes was not evaluated.

The 2 factors associated with an increased IRM on multivariate analysis were dialysis requirements and CNS status. With end-organ failure, dialysis requirement serves as a proxy to multiple systemic processes including, but not limited to TLS, tumor burden, and drug toxicity. Similar to the results observed in acute kidney injury during acute myeloid leukemia induction therapy [22], the survival of patients requiring hemodialysis in our cohort was favorable only for those achieving CR1, contrary to the 5 patients not achieving this response and who died prior to day +60 . The influence of CNS status on long-term outcomes and mortality in ALL has been well described in the literature $[23,24]$. The role of this factor and its relationship with IRM has been far less studied, but possible explanations include greater leukemic burden and the proliferation of far more aggressive leukemic phenotypes.

We acknowledge that the retrospective nature, sample size, and lack of molecular profile assessment in our study are important limitations; nevertheless, the particularities of ALL in Latin America urges the need to analyze setting-specific data to promote the adaptation of evidence-based medicine in the current clinical practice and resources of hematologists. This and other real-world studies set the base for further research in the field of adult ALL in LMICs. 
In conclusion, this study supports previous data showing that in Latin America, adult ALL patients treated with intensive induction regimens have higher IRM and complication rates than those in high-income countries. A higher mortality rate in our population was mainly related to patients presenting with end-stage organ disease and higher tumor burden at diagnosis. In the future, prospective studies that include pediatric-appropriate regimens and molecular diagnoses are warranted to further analyze possible factors that can predict IRM in this particular group of patients.

\section{Authors' Disclosure of Potential Conflict of Interest}

No potential conflicts of interest relevant to this article were reported. 


\section{REFERENCES}

1. Terwilliger T, Abdul-Hay M. Acute lymphoblastic leukemia: a comprehensive review and 2017 update. Blood Cancer J 2017;7:e577.

2. Larson RA, Dodge RK, Burns CP, et al. A five-drug remission induction regimen with intensive consolidation for adults with acute lymphoblastic leukemia: cancer and leukemia group B study 8811. Blood 1995;85:2025-37.

3. Wheeler K, Chessells JM, Bailey CC, Richards SM. Treatment related deaths during induction and in first remission in acute lymphoblastic leukaemia: MRC UKALL X. Arch Dis Child 1996;74:101-7.

4. Ramos C, Rozen E, León M, et al. Results of treatment of acute lymphoblastic leukemia in two cohorts of Mexican patients. Rev Med Chile 2011;139:1135-42.

5. Fernandes da Silva Junior W, Medina AB, Yamakawa PE, Buccheri V, Velloso EDRP, Rocha

V. Treating adult acute lymphoblastic leukemia in Brazil-increased early mortality using a German multicenter acute lymphoblastic leukemia-based regimen. Clin Lymphoma Myeloma Leuk 2018;18:e255-e9.

6. Chessells JM, Hall E, Prentice HG, Durrant J, Bailey CC, Richards SM. The impact of age on outcome in lymphoblastic leukaemia; MRC UKALL X and XA compared: a report from the MRC Paediatric and Adult Working Parties. Leukemia 1998;12:463-73.

7. Roberts KG. Genetics and prognosis of ALL in children vs adults. Hematology Am Soc Hematol Educ Program 2018;2018:137-45.

8. Pichler H, Reismüller B, Steiner M, et al. The inferior prognosis of adolescents with acute lymphoblastic leukaemia (ALL) is caused by a higher rate of treatment-related mortality and not an increased relapse rate--a population-based analysis of 25 years of the Austrian ALL-BFM (BerlinFrankfurt-Münster) Study Group. Br J Haematol 2013;161:556-65.

9. Gupta S, Antillon FA, Bonilla M, et al. Treatment-related mortality in children with acute lymphoblastic leukemia in Central America. Cancer 2011;117:4788-95.

10. Slats AM, Egeler RM, van der Does-van den Berg A, et al. Causes of death--other than progressive leukemia--in childhood acute lymphoblastic (ALL) and myeloid leukemia (AML): the Dutch Childhood Oncology Group experience. Leukemia 2005;19:537-44.

11. Oken MM, Creech RH, Tormey DC, et al. Toxicity and response criteria of the Eastern Cooperative Oncology Group. Am J Clin Oncol 1982;5:649-55.

12. Swerdlow SH, Campo E, Pileri SA, et al. The 2016 revision of the World Health Organization classification of lymphoid neoplasms. Blood 2016;127:2375-90.

13. Brown PA, Wieduwilt M, Logan A, et al. Guidelines Insights: Acute Lymphoblastic Leukemia, Version 1.2019. J Natl Compr Canc Netw 2019;17:414-23. 
14. Kantarjian HM, O'Brien S, Smith TL, et al. Results of treatment with hyper-CVAD, a doseintensive regimen, in adult acute lymphocytic leukemia. J Clin Oncol 2000;18:547-61.

15. Arteaga-Ortiz L, Buitrón-Santiago N, Rosas-López A, et al. Acute lymphoblastic leukemia: experience in adult patients treated with hyperCVAD and 0195 Protocol, at the Instituto Nacional de Ciencias Médicas y Nutrición Salvador Zubirán. Cohort 2003-2007. Rev Invest Clin 2008;60:459-69.

16. Ribera JM, Oriol A. Acute lymphoblastic leukemia in adolescents and young adults. Hematol Oncol Clin North Am 2009;23:1033-42, vi.

17. Thomas DA, O'Brien S, Kantarjian HM. Monoclonal antibody therapy with rituximab for acute lymphoblastic leukemia. Hematol Oncol Clin North Am 2009;23:949-71, v.

18. Jaime-Pérez JC, Jiménez-Castillo RA, Herrera-Garza JL, Gutiérrez-Aguirre H, Marfil-Rivera LJ, Gómez-Almaguer D. Survival rates of adults with acute lymphoblastic leukemia in a low-income population: a decade of experience at a single institution in Mexico. Clin Lymphoma Myeloma Leuk 2017;17:60-8.

19. Lepe-Zuniga JL, Ramirez-Nova V. Elements associated with early mortality in children with B cell acute lymphoblastic leukemia in Chiapas, Mexico: a case-control study. J Pediatr Hematol Oncol 2019;41:1-6.

20. Mariette C, Tavernier E, Hocquet D, et al. Epidemiology of invasive fungal infections during induction therapy in adults with acute lymphoblastic leukemia: a GRAALL-2005 study. Leuk Lymphoma 2017;58:586-93.

21. Lehrnbecher $T$. Infectious complications in acute lymphoblastic leukemia: individually tailored prevention and treatment. Pharm Unserer Zeit 2012;41:228-33.

22. Lahoti A, Kantarjian H, Salahudeen AK, et al. Predictors and outcome of acute kidney injury in patients with acute myelogenous leukemia or high-risk myelodysplastic syndrome. Cancer 2010;116:4063-8.

23. Thomas X, Le QH. Central nervous system involvement in adult acute lymphoblastic leukemia. Hematology 2008;13:293-302.

24. Lazarus HM, Richards SM, Chopra R, et al. Central nervous system involvement in adult acute lymphoblastic leukemia at diagnosis: results from the international ALL trial MRC UKALL XII/ECOG E2993. Blood 2006;108:465-72. 


\section{Tables}

Table 1. Patients baseline characteristics grouped by AYA and non-AYA.

\begin{tabular}{|c|c|c|}
\hline Characteristics & $\operatorname{AYA}(\mathrm{N}=112)$ & Non-AYA $(\mathrm{N}=55)$ \\
\hline Male sex, N (\%) & $59(52.7)$ & $26(47.3)$ \\
\hline Age, median (range) & $22(16-39)$ & $51(40-70)$ \\
\hline \multicolumn{3}{|l|}{ Socioeconomic status, N (\%) } \\
\hline Low-income & $111(99.1)$ & $47(85.5)$ \\
\hline Middle-income & $0(0)$ & $3(5.4)$ \\
\hline High-income & $1(0.9)$ & $5(9.1)$ \\
\hline $\mathrm{ECOG} \leq 2, \mathrm{~N}(\%)$ & $109(97.3)$ & $52(94.5)$ \\
\hline Comorbidities, N (\%) & $45(40.2)$ & $36(65.5)$ \\
\hline $\mathrm{DM}$ & $2(1.8)$ & $15(27.3)$ \\
\hline HTN & $4(3.6)$ & $9(16.4)$ \\
\hline Obesity & $21(18.8)$ & $15(27.3)$ \\
\hline \multicolumn{3}{|l|}{ ALL phenotype, $\mathrm{N}(\%)$} \\
\hline B-cell leukemia & $109(97.3)$ & $54(98.2)$ \\
\hline Pre-B & $102(93.6)$ & $54(100)$ \\
\hline Mature B & $6(5.5)$ & $0(0)$ \\
\hline Pro-B & $1(0.9)$ & $0(0)$ \\
\hline T-cell leukemia & $3(2.7)$ & $1(1.8)$ \\
\hline Cytogenetic abnormalities $^{\mathrm{a})}, \mathrm{N}(\%)$ & $28(25.5)$ & $20(38.5)$ \\
\hline Philadelphia chromosome & $17(15.5)$ & $12(23.1)$ \\
\hline MLL rearrangements & $1(0.9)$ & $1^{\mathrm{c})}(1.9)$ \\
\hline Hypodiploidy & $0(0)$ & $2(3.9)$ \\
\hline Complex karyotype & $5^{b)}(4.6)$ & $1(1.9)$ \\
\hline Others & $8(7.3)$ & $5(9.6)$ \\
\hline CNS involvement, $\mathrm{N}(\%)$ & $13(11.6)$ & $6(10.9)$ \\
\hline
\end{tabular}




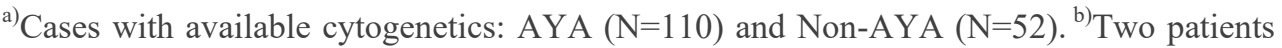
$\mathrm{Ph}+$ and one with $M L L$ rearrangement in addition to complex karyotype. ${ }^{\text {c) }} \mathrm{Patient} \mathrm{Ph}+$ in addition to $M L L$ rearrangement.

Abbreviations: ALL, acute lymphoblastic leukemia; AYA, adolescent and young adult; CNS, central nervous system; DM, diabetes mellitus; ECOG, Eastern Cooperative Oncology Group; HTN, hypertension; MLL, mixed-lineage leukemia; Ph+, Philadelphia positive. 
Table 2. Induction-related complications.

\begin{tabular}{l|c}
\hline Complications & $\mathrm{N}=152$ \\
\hline Infectious, N (\%) & $146(87.4)$ \\
Bloodstream infection & $45(30.8)$ \\
UTI & $23(15.8)$ \\
Pneumonia & $55(37.7)$ \\
Skin and soft tissue & $33(22.6)$ \\
C. difficile colitis & $10(6.9)$ \\
IFI & $29(19.9)$ \\
Metabolic, N (\%) & $70(46.1)$ \\
Hipertransaminasemia & $22(31.4)$ \\
Dialysis requirement & $7(10.0)$ \\
TLS & $41(58.6)$ \\
Hematologic, N (\%) & $5(27.8)$ \\
DiC & $7(38.9)$ \\
Abrombosis & $6(33.3)$ \\
\hline
\end{tabular}

Abbreviations: DIC, disseminated intravascular coagulation; IFI, invasive fungal infections; TLS, tumor lysis syndrome; UTI, urinary tract infection. 
Table 3. Factors related to a decreased overall survival after induction.

\begin{tabular}{|c|c|c|c|}
\hline & $\begin{array}{l}\text { Univariate analysis } \\
\text { OS Day+60 (\%) }\end{array}$ & $P$ & $\begin{array}{c}\text { Multivariate analysis } \\
\text { HR }(95 \% \mathrm{CI})\end{array}$ \\
\hline Central nervous system involvement & & & CNS-3: $3.078(0.81-11.67)$ \\
\hline CNS-3 vs. CNS- 2 vs. CNS- 1 & $73.7 \%$ vs. $37.5 \%$ vs. $92.7 \%$ & $<0.001$ & CNS-2: $10.10(2.67-38.18)$ \\
\hline TLS & $75.3 \%$ vs. $85.12 \%$ & 0.005 & - \\
\hline DIC & $66.7 \%$ vs. $89.4 \%$ & 0.037 & - \\
\hline Shock & $63.2 \%$ Vs. $93.8 \%$ & $<0.001$ & - \\
\hline Bloodstream infection & $83.1 \%$ vs. $95.3 \%$ & 0.020 & - \\
\hline Dialysis requirement & $28.6 \%$ vs. $91.0 \%$ & $<0.001$ & $9.224(2.45-33.72)$ \\
\hline
\end{tabular}

Abbreviations: CNS, central nervous system; DIC, disseminated intravascular coagulation; OS, overall survival; TLS, tumor lysis synd 
A

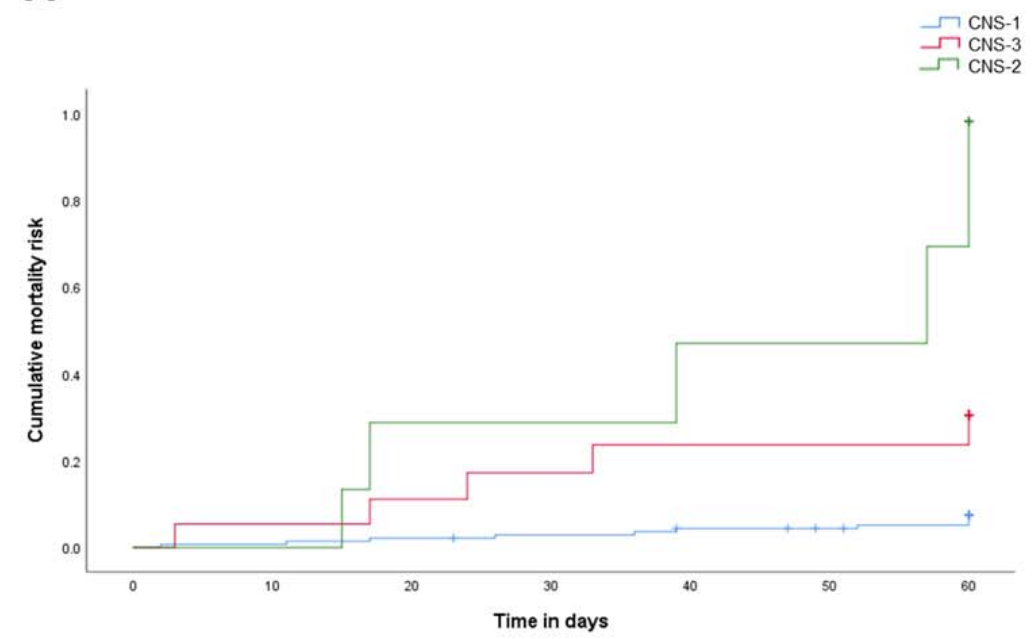

B

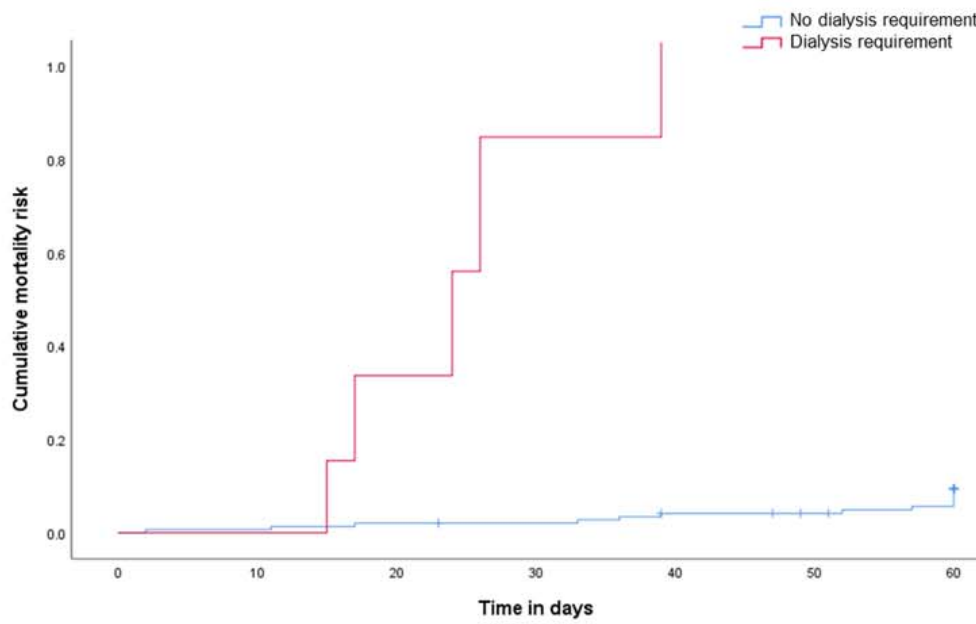

Fig. 1. Cumulative mortality risk related to (A) central nervous system status and (B) dialysis requirement. 